Artigo de Revisão 


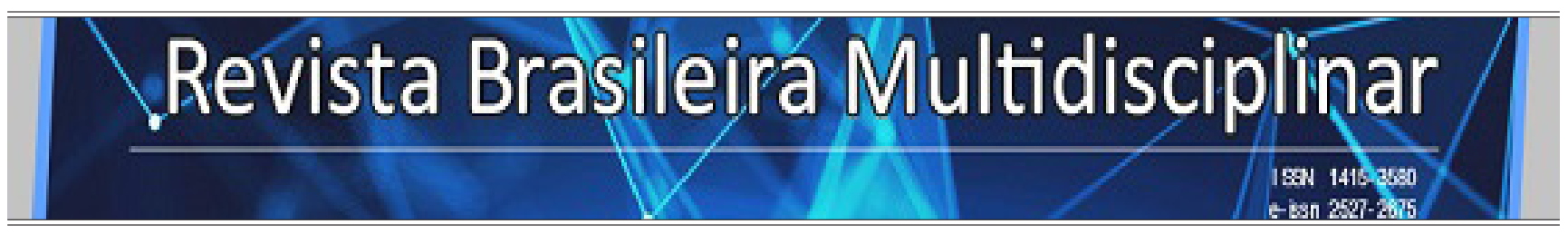

http://revistarebram.com/index.php/revistauniara

\section{Análise da produção científica sobre a tuberculose em portugal: revisão INTEGRATIVA DA LITERATURA}

Giovanna Mendes da Silva Maia*; Ana Luísa dos Santos**; Rogério Manuel Clemente Rodrigues ${ }^{\star * *}$; Pedro Fredemir Palha ${ }^{* * *}$; Ana Carolina Scarpel Moncaio ${ }^{\star * * * *}$.

* Enfermeira. Graduada pela Universidade Federal do Amazonas/Escola de Enfermagem de Manaus.

** Doutora em Antropologia. Docente do Departamento de Ciências da Vida e Investigadora do Centro de Investigação em Antropologia e Saúde (CIAS) da Universidade de Coimbra.

${ }_{* * *}^{*}$ Doutor em Enfermagem. Docente da Escola Superior de Enfermagem de Coimbra, Portugal.

**** Doutor em Enfermagem. Docente da Universidade de São Paulo/Escola de Enfermagem de Ribeirão Preto.

***** Doutora em Enfermagem. Docente da Universidade Federal de Catalão, Brasil.

*Autor para correspondência e-mail: carolina_scarpel@ufg.br

\section{Palavras-chave}

Mycobacterium Tuberculosis Tuberculose

Portugal

\section{KeYWORDS}

Mycobacterium Tuberculosis Tuberculosis

Portugal

\section{RESUMO}

Em 2017, foram notificados 1741 casos de tuberculose, dos quais 1607 são novos. Dados que representam uma estimativa da taxa de incidência de 15,6 por 100.000 habitantes, mantendo-se uma redução de $5 \%$ ao ano. Comparativamente ao início do milênio, que possuía taxa de incidência de $40 \%$, estes resultados indicam uma evolução positiva do país no controle da tuberculose. O objetivo deste estudo foi analisar a produção científica sobre a tuberculose em Portugal. Trata-se de uma Revisão Integrativa da Literatura, com pesquisa de artigos atendendo a critérios pré-definidos, considerando o período temporal entre 2006 a 2017, acedidos por meio das bases de dados PubMed e CINAHL. Foram identificados 23 artigos cujos resultados são diversificados, fornecendo um panorama sobre a realidade nacional, especialmente no que concerne a prevalência de casos, fatores de risco e resultados do tratamento, multirresistência e testes de rastreio em profissionais de saúde. Os resultados podem ser úteis para a melhoria de medidas tanto dos programas de luta contra a tuberculose, quanto das outras esferas organizacionais responsáveis por fornecer ações de saúde.

\begin{abstract}
ANALysis OF SCIENTIFIC PRODUCTION ON TUBERCULOSIS IN PORTUGAL: AN INTEgRATIVE LITERATURE REVIEW

In 2017, 1.741 tuberculosis cases were notified, of which 1.607 are new. Data that represent an estimate of the incidence rate of 15,6 per 100.000 inhabitants, maintaining a reduction of $5 \%$ per year. Compared to the beginning of the millennium, which had an incidence rate of $40 \%$, these results indicate a positive evolution of the country in the control of the tuberculosis disease. The aim of this study was to analyze the scientific production on tuberculosis in Portugal. This is an Integrative Literature Review with research of articles meeting pre-defined criteria, considering the time period between 2006 and 2017, accessed through PubMed and CINAHL databases. A total of 23 articles were identified whose results are diverse, providing an overview of the national reality, especially regarding incidence of cases, risk factors and treatment outcomes, multidrug resistance and screening tests in health professionals. The results can be useful for improving measures of both tuberculosis control programs and other organizational spheres responsible for delivering health actions.
\end{abstract}




\section{INTRODUÇÃo}

Doença milenar, a Tuberculose (TB) é considerada uma doença grave desde as primeiras menções em antigas literaturas. Em 2015, estima-se que ocorreram 10,4 milhões de casos novos, dos quais 1,2 milhões destes indivíduos são portadores do Vírus da Imunodeficiência Humana (VIH) sendo 5,9 milhões homens, 3,5 milhões mulheres e cerca de 1 milhão entre crianças (World Health Organization, 2016). No mesmo ano, esteve no ranking das 10 doenças que mais ocasionaram óbitos no mundo, prevalecendo sobre o VIH e a Síndrome da Imunodeficiência Adquirida (SIDA), tornando-se uma das principais causas de morte por doença infecciosa e se caracterizando como um grande problema de saúde pública mundial (WORLD HEALTH ORGANIZATION, 2016).

Segundo o European Centre for Disease Prevention and Control (ECDPC), desde o início século XXI, na Europa, o número estimado de casos de TB tem diminuído progressivamente, apresentando uma média anual estimadade declínio de 4,3\% entre 2007 e 2016, com 4,6\% entre 2014 e 2016. Os dados concernentes ao ano de 2016 são de 290.000 novos casos de TB e reincidências, equivalendo a 31,6\% casos por 100.000 habitantes e representando 3\% do total dos eventos globais. De acordo com a fonte, durante esse ano, o número absoluto de casos reduziu 13.000 se comparado ao ano anterior, podendo ser identificado por meio das taxas de notificação (ECDPC, 2018).

Para Portugal, o ECDPC (2018) também afirma que, no ano de 2016, ocorreram 1836 casos notificados de TB, correspondendo a uma taxa de incidência de 17,8 por 10.000 habitantes, sendo 1123 os casos confirmados. Relativamente a 2017, foram notificados 1741 casos, dos quais 1607 são novos casos (PORTUGAL, 2017). Estes dados representam uma estimativada taxa de notificação de 16,9 por 100.000 habitantes e uma taxa de incidência de 15,6 por 100.000 habitantes, mantendo-se uma redução das taxas de $5 \%$ ao ano. Comparativamente ao início do milénio, o qual possuía taxa de incidência de $40 \%$, estes resultados indicam uma evolução positiva do país no controle da TB (PORTUGAL, 2017).

Verifica-se a concentração de casos nos distritos de Lisboa e Porto, grandes centros urbanos, cujo impacto social da crise económica do país ocasionou o aumento do desemprego, desigualdade económica, pobreza, deterioração das condições de vida da população e sobrelotação dos domicílios. Tais fatores socioeconómicos, além dos estilos de vida e consumo de drogas lícitas e ilícitas pela população podem afetar negativamente as conquistas da saúde pública, principalmente relacionadas ao controle da TB, pois reduzem as defesas naturais contra esta doença e, eventualmente, aumentam o número de casos e interferem em tratamentos em curso (COUCEIRO; SANTANA; NUNES, 2011).

Aliados aos fatores supracitados, as medidas de prevenção, diagnóstico e tratamento, assim como de controle e avaliação dos resultados obtidos no combate à TB, se não aplicada de forma eficaz contribuem para o descontrole da incidência desta (PORTUGAL, 1995). A atuação de forma organizada e integrada torna-se necessária para assegurar o acesso da população a um serviço de qualidade e assegurar o controle da TB.

Convergindo com as estratégias Stop-TB (2006) e End-TB (2015) propostas pela Organização Mundial de Saúde (OMS), a redução dos índices de TB exige um ação harmônica entre governo, investigadores, profissionais de saúde, organizações da sociedade civil e comunidades de pessoas infectadas.

Com o intuito de cooperar com os desafios de controle que os serviços de saúde enfrentam, propôs-se a investigação com o objetivo de analisar a produção científica sobre a TB em Portugal. Para tal, foi elaborada a seguinte questão orientadora desta revisão integrativa: "Qual a produção científica sobre tuberculose em Portugal?".

\section{Metodologia}

Trata-se de uma revisão integrativa da literatura, que sintetiza a literatura empírica e teórica passada 
para fornecer uma compreensão mais abrangente de um fenômeno particular por meio do estabelecimento de conclusões de vários estudos (WHITTEMORE; KNAFL, 2005).

Para a elaboração da presente revisão integrativa, os seguintes processos metodológicos foram adotados: definição da questão de investigação; estabelecimento da seleção da amostra; definição dos dados a serem extraídos; avaliação das pesquisas incluídas; interpretação dos resultados e apresentação da revisão integrativa (WHITTEMORE; KNAFL, 2005).

Para a seleção dos artigos foi realizada extensa pesquisa bibliográfica nas duas bases de dados Pubmed e CINAHL. Os descritores para o levantamento dos artigos foram selecionados a partir da terminologia em saúde consultada no Medical Subject Heading (MeSH); sendo eles: Tuberculosis AND Portugal.

Os critérios de inclusão definidos foram artigos originais, com resumos disponíveis nas bases de dados selecionadas, no idioma português e inglês, no período compreendido entre 2006 e 2017. Revisões de literatura, dissertações de mestrado, teses de doutorado, estudos de caso, estudos moleculares e genéticos, artigos com dados ou maioria dos dados anteriores a 2006 foram excluídos.

O motivo pelo qual se optou pelo ano de 2006 está relacionado ao lançamento da estratégia Stop-TB pela OMS, a qual visava à diminuição dos casos de TB até o ano de 2015. Portanto, para verificação da implementação das medidas sugeridas pela estratégia, tem-se como limiar o ano de 2006.

Na busca realizada, foram identificados 16 estudos na base de dados CINAHL e 757 na PubMed (Figura 1). Após a eliminação dos itens duplicados e leitura dos textos na íntegra, segundo pertinência e consistência do conteúdo e aplicação dos critérios de inclusão e exclusão, foram excluídos 745 artigos (14 da base de dados CINAHL e 731 da PubMed). Assim, após esta fase, iniciou-se a análise de 23 estudos completos, sendo dois da CINAHL e 21 da PubMed.

Elaborou-se para organização da análise das produções (Figura 1) que atenderam aos critérios de inclusão contendo os itens: autores, ano de publicação, título, base de dados e publicação periódica. Embora os artigos apresentassem aspectos diversificados relacionados à $\mathrm{TB}$, foi possível identificar nos resultados pontos convergentes que culminaram na classificação dos mesmos em três categorias: "Prevalência de casos, fatores de risco e resultados do tratamento", "Tuberculose multirresistente" e "Testes de rastreio em profissionais de saúde".

Em virtude da natureza da pesquisa, não foi necessário submeter o projeto para apreciação da Comissão de Ética. 
Figura 1- Fluxograma de seleção dos artigos.
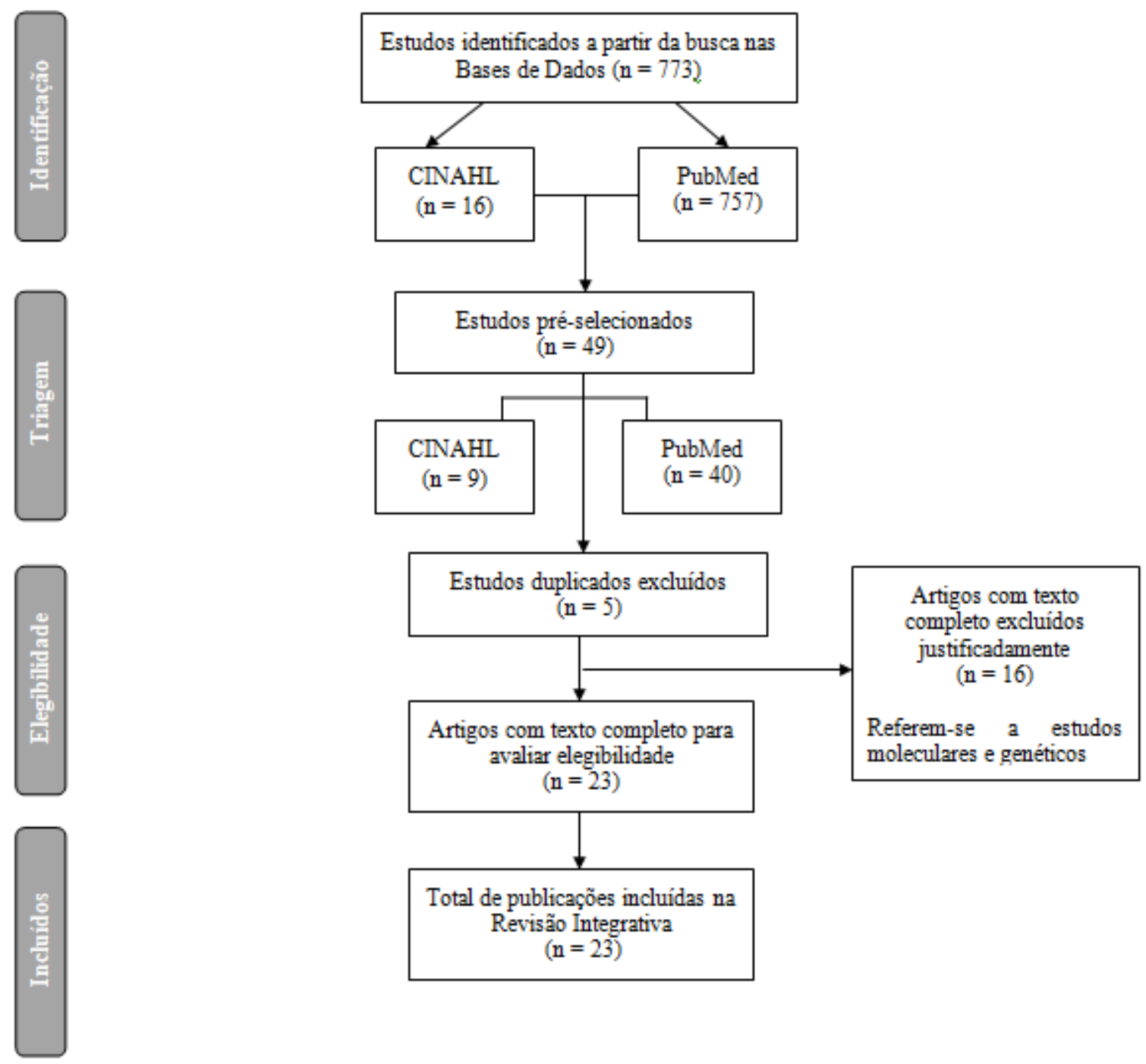

Fonte: Moher et al., 2009.

\section{Resultados e Discussão}

Os artigos selecionados estavam inseridos em oito publicações periódicas que apresentavam uma diversidade em relação à área de interesse. Os focos das publicações periódicas centravam-se individualmente em epidemiologia e infecções, medicina ocupacional, pneumologia, TB, doenças respiratórias e micobacteriologia. Este fato demonstra que a produção científica na temática da TB não se limita a publicações periódicas especializadas em infecciologia, mas também na área da medicina ocupacional. Cabe salientar a utilização da abordagem quantitativa na totalidade da amostra de artigos selecionados da revisão.

A distribuiçãos dos artigos selecionados para o presente estudo selecionados segundo autores, ano de publicação, título, base de dados e revista encontram-se no Quadro 1. 
Quadro 1- Distribuição dos artigos selecionados segundo autores, ano de publicação, título, base de dados e revista.

\begin{tabular}{|c|c|c|c|c|c|}
\hline $\mathbf{N}^{\circ}$ & Autores & Ano & Título & $\begin{array}{c}\text { Base de } \\
\text { dados }\end{array}$ & Revista \\
\hline 1 & Areias,Briz e Nunes & 2015 & $\begin{array}{l}\text { Pulmonary tuberculosis space-time } \\
\text { clustering and spatial } \\
\text { variation in temporal trends in Portu- } \\
\text { gal, 2000-2010: an } \\
\text { updated analysis }\end{array}$ & CINAHL & Epidemiology \& Infection \\
\hline 2 & $\begin{array}{l}\text { Torres Costa, Silva, } \\
\text { Ringshausen e Nie- } \\
\text { nhaus }\end{array}$ & 2011 & $\begin{array}{l}\text { Screening for tuberculosis and predic- } \\
\text { tion of } \\
\text { disease in Portuguese healthcare work- } \\
\text { ers }\end{array}$ & CINAHL & $\begin{array}{l}\text { Journal of Occupational } \\
\text { Medicine and Toxicology }\end{array}$ \\
\hline 3 & $\begin{array}{l}\text { Viveiros, Mota, Brinca, } \\
\text { Carvalho e Duarte }\end{array}$ & 2013 & $\begin{array}{l}\text { Adesão ao rastreio e tratamento da } \\
\text { tuberculose em doentes } \\
\text { infetados com o vírus da imunodefi- } \\
\text { ciência humana }\end{array}$ & PubMed & $\begin{array}{l}\text { Revista Portuguesa de } \\
\text { Pneumologia }\end{array}$ \\
\hline 4 & $\begin{array}{l}\text { Ladeira, Correia, Dias, } \\
\text { Gaio, Carvalho, Carva- } \\
\text { lho e Duarte }\end{array}$ & 2014 & $\begin{array}{l}\text { Confirming the diagnosis of tuberculo- } \\
\text { sis in children in } \\
\text { Northern Portugal }\end{array}$ & PubMed & $\begin{array}{l}\text { The International Journal } \\
\text { of Tuberculosis and Lung } \\
\text { Disease }\end{array}$ \\
\hline 5 & $\begin{array}{l}\text { Mendes, Gaio, Reis e } \\
\text { Duarte }\end{array}$ & 2013 & $\begin{array}{l}\text { Contact screening in tuberculosis: can } \\
\text { we identify } \\
\text { those with higher risk? }\end{array}$ & PubMed & $\begin{array}{l}\text { European Respiratory } \\
\text { Journal }\end{array}$ \\
\hline 6 & \begin{tabular}{|l} 
Guimarães, Oliveira, \\
Teixeira, Gaio e Duarte
\end{tabular} & 2015 & Delay diagnosis of tuberculosis & PubMed & $\begin{array}{l}\text { Revista Portuguesa de } \\
\text { Pneumologia }\end{array}$ \\
\hline 7 & $\begin{array}{l}\text { Meireles, Gaio e Duar- } \\
\text { te }\end{array}$ & 2014 & $\begin{array}{l}\text { Factors influencing tuberculosis } \\
\text { screening } \\
\text { in healthcare workers in Portugal }\end{array}$ & PubMed & $\begin{array}{l}\text { European Respiratory } \\
\text { Journal }\end{array}$ \\
\hline 8 & $\begin{array}{l}\text { Reina, Silva, Gaio, Cor- } \\
\text { reia e Duarte }\end{array}$ & 2015 & $\begin{array}{l}\text { HIV screening in tuberculosis patients } \\
\text { in the } \\
\text { northern region of Portugal }\end{array}$ & PubMed & $\begin{array}{l}\text { The International Journal } \\
\text { of Tuberculosis and Lung } \\
\text { Disease }\end{array}$ \\
\hline 9 & $\begin{array}{l}\text { Castro, Mendes, Freitas } \\
\text { e Roxo }\end{array}$ & 2015 & $\begin{array}{l}\text { Incidence and risk factors of major } \\
\text { toxicity associated to first-line antitu- } \\
\text { berculosis drugs for latent and active } \\
\text { tuberculosis during a period of } 10 \\
\text { years }\end{array}$ & PubMed & $\begin{array}{l}\text { Revista Portuguesa de } \\
\text { Pneumologia }\end{array}$ \\
\hline 10 & $\begin{array}{l}\text { Sousa, Oliveira, Gomes, } \\
\text { Gaio e Duarte }\end{array}$ & 2016 & $\begin{array}{l}\text { Longitudinal clustering of tuberculosis } \\
\text { incidence and predictors } \\
\text { for the time profiles: the impact of HIV }\end{array}$ & PubMed & $\begin{array}{l}\text { The International Journal } \\
\text { of Tuberculosis and Lung } \\
\text { Disease }\end{array}$ \\
\hline 11 & $\begin{array}{l}\text { Macedo, Antunes, } \\
\text { Villar e Portugal }\end{array}$ & 2012 & $\begin{array}{l}\text { Multidrug and extensively drug-resis- } \\
\text { tant tuberculosis } \\
\text { in Lisbon and Vale do Tejo, Portugal, } \\
\text { from } 2008 \text { to } 2010\end{array}$ & PubMed & $\begin{array}{l}\text { International Journal of } \\
\text { Mycobacteriology }\end{array}$ \\
\hline
\end{tabular}


Quadro 1- Distribuição dos artigos selecionados segundo autores, ano de publicação, título, base de dados e revista. (cont.)

\begin{tabular}{|c|c|c|c|c|c|}
\hline 12 & $\begin{array}{l}\text { Oliveira, Gaio, Villar e } \\
\text { Duarte }\end{array}$ & 2013 & $\begin{array}{l}\text { Predictors of treatment outcome in } \\
\text { multidrug-resistant tuberculosis in } \\
\text { Portugal }\end{array}$ & PubMed & $\begin{array}{l}\text { European Respiratory } \\
\text { journal }\end{array}$ \\
\hline 13 & $\begin{array}{l}\text { Cordeiro Costa, Olivei- } \\
\text { ra, Baía, Gaio, Correia- } \\
\text {-Neves e Duarte }\end{array}$ & 2016 & $\begin{array}{l}\text { Prevalence and factors associated with } \\
\text { diabetes mellitus among tuberculosis } \\
\text { patients: a nationwide cohort }\end{array}$ & PubMed & $\begin{array}{l}\text { European Respiratory } \\
\text { Journal }\end{array}$ \\
\hline 14 & $\begin{array}{l}\text { Eufrásio, Alcobia e } \\
\text { Correia }\end{array}$ & 2016 & $\begin{array}{l}\text { Pulmonary tuberculosis epidemiology } \\
\text { in Coimbra's District (2000---2011): } \\
\text { Information is essential to understand } \\
\text { high risk groups }\end{array}$ & PubMed & $\begin{array}{l}\text { Revista Portuguesa de } \\
\text { Pneumologia }\end{array}$ \\
\hline 15 & $\begin{array}{l}\text { Eufrásio, Alcobia e } \\
\text { Correia }\end{array}$ & 2017 & $\begin{array}{l}\text { Pulmonary tuberculosis:Resistance pat- } \\
\text { tern to first lineanti-tuberculosis drugs } \\
\text { in theCoimbra District, } 2000-2011\end{array}$ & PubMed & $\begin{array}{l}\text { Revista Portuguesa de } \\
\text { Pneumologia }\end{array}$ \\
\hline 16 & $\begin{array}{l}\text { Melo, Baía, Gaio e } \\
\text { Duarte }\end{array}$ & 2016 & Silicosis, tuberculosis timebomb? & PubMed & $\begin{array}{l}\text { Revista Portuguesa de } \\
\text { Pneumologia }\end{array}$ \\
\hline 17 & $\begin{array}{l}\text { Franco, Sousa, Go- } \\
\text { mes, Oliveira, Gaio e } \\
\text { Duarte }\end{array}$ & 2016 & $\begin{array}{l}\text { Social profile of the highesttuberculosis } \\
\text { incidence areasin Portugal }\end{array}$ & PubMed & $\begin{array}{l}\text { Revista Portuguesa de } \\
\text { Pneumologia }\end{array}$ \\
\hline 18 & $\begin{array}{l}\text { Bras, Gomes, Filipe,- } \\
\text { Sousa e Nunes }\end{array}$ & 2014 & $\begin{array}{l}\text { Trends, seasonality and forecasts of } \\
\text { pulmonary tuberculosis in } \\
\text { Portugal }\end{array}$ & PubMed & $\begin{array}{l}\text { The International Journal } \\
\text { of Tuberculosis and Lung } \\
\text { Disease }\end{array}$ \\
\hline 19 & $\begin{array}{l}\text { Redondo, Carvalho, } \\
\text { Correia e Duarte }\end{array}$ & 2017 & $\begin{array}{l}\text { Tuberculosis Among Portuguese Living } \\
\text { Abroad }\end{array}$ & PubMed & $\begin{array}{l}\text { Archivos de Bronconeu- } \\
\text { mología }\end{array}$ \\
\hline 20 & $\begin{array}{l}\text { Dias, Gaio, Sousa, } \\
\text { Abranches, Gomes, Oli- } \\
\text { veira, Correia-Neves, } \\
\text { Ferreira e Duarte }\end{array}$ & 2017 & $\begin{array}{l}\text { Tuberculosis among the homeless: } \\
\text { should we change the } \\
\text { strategy? }\end{array}$ & PubMed & $\begin{array}{l}\text { The International Journal } \\
\text { of Tuberculosis and Lung } \\
\text { Disease }\end{array}$ \\
\hline 21 & $\begin{array}{l}\text { Paulino, Martins, Ma- } \\
\text { chado, Gomes, Gaio e } \\
\text { Duarte }\end{array}$ & 2016 & $\begin{array}{l}\text { Tuberculosis in native- and for- } \\
\text { eign-born populations in Portugal }\end{array}$ & PubMed & $\begin{array}{l}\text { The International Journal } \\
\text { of Tuberculosis and Lung } \\
\text { Disease }\end{array}$ \\
\hline 22 & $\begin{array}{l}\text { Pacheco, Silva, Oliveira, } \\
\text { Carvalho,Correia e } \\
\text { Duarte }\end{array}$ & 2015 & $\begin{array}{l}\text { Tuberculosis retreatment in Northern } \\
\text { Portugal }\end{array}$ & PubMed & $\begin{array}{l}\text { Revista Portuguesa de } \\
\text { Pneumologia }\end{array}$ \\
\hline 23 & $\begin{array}{l}\text { Josaphat, Gomes Dias, } \\
\text { Salvador, Resende e } \\
\text { Duarte }\end{array}$ & 2014 & $\begin{array}{l}\text { Tuberculosis: Which patients do not } \\
\text { identify their } \\
\text { contacts? }\end{array}$ & PubMed & $\begin{array}{l}\text { Revista Portuguesa de } \\
\text { Pneumologia }\end{array}$ \\
\hline
\end{tabular}

Fonte: elaborado pelos autores.

Os resultados e discussão apresentados a seguir seguem a análise das categorias anteriormente citadas: "prevalência de casos, fatores de risco e resultados do tratamento", "tuberculose multirresistente" e "testes de rastreio em profissionais de saúde”, enfatizando sempre que se julgue necessário com o reforço de estudos consultados para além dos selecionados na presente revisão integrativa da literatura.

\section{Prevalência de Casos, fatores de risco e resultados do tratamento}


Os dados presentes nos artigos consultados indicam que, apesar do número decrescente de casos ao longo do tempo, a susceptibilidade para contrair a TB correlaciona-se com a infecção pelo VIH, desemprego, uso de drogas lícitas e ilícitas, viver em situação de rua, presença de comorbidades como Diabetes mellitus (DM) e hepatite $\mathrm{C}$, fatores estes que corroboram para aumento do número de casos (SOUSA, 2016; EUFRÁSIO, 2016).

Em relação às áreas com maior incidência do país, destacam-se Lisboa, Porto e Península de Setúbal, estando os altos índices fortemente ligados a fatores socioeconómicos, como aumento das taxas de desemprego e imigração (AREIAS, 2015; FRANCO, 2016). Evidenciou-se que Lisboa e Porto são as localidades contribuintes para picos sazonais da TB, devido à sua densidade demográfica, apresentando como alvo a população masculina e adulta com idade entre 25 e 54 anos (BRAS, 2014). Neste contexto, faz-se necessário que as ações de controle da TB estejam adaptadas para a realidade de cada região para a redução da morbimortalidade e que sejam adotadas estratégias para a redução da desigualdade social, uma vez que a TB continua intimamente relacionada com as condições de vida da população (SAN PEDRO; MAGALHÃES DE OLIVEIRA, 2013).

Colocando em perspectiva a população feminina portuguesa, possuir emprego, grau académico e utilizar transporte privado para chegar aos serviços de saúde foram associados com maior tempo de espera até a conclusão do diagnóstico de TB, sendo duas vezes maior em relação aos homens. Mais pesquisas precisam ser feitas para identificar os motivos pelo qual esta população em particular, dispondo de status social melhorado, apresenta demora no diagnóstico (GUIMARÃES, 2015).

Considera-se ainda, de acordo com Paulino et al. (2016), que a cultura tabágica e alcoólica da população portuguesa é um fator de risco significativo para a TB, assim como o desemprego. Segundo Molina, Happel, Zhang, Kolls e Nelson (2010), o consumo constante e excessivo de álcool produz efeito depressor sobre os componentes imunológicos do sistema respiratório e associado ao consumo de tabaco, cuja fumaça provoca a redução do clearance mucociliar do trato respiratório, aumentando a aderência das bactérias e rompendo o epitélio protetor, propicia que o individuo esteja exposto ao risco de contrair TB, como referido por Rabahi (2012).

Ainda em relação a fatores culturais, a presença dos imigrantes nos país apresenta dados diferenciados dos observados na população portuguesa. Os fatores de risco evidenciados foram: doentes nascidos no exterior eram mais jovens e menos propensos a serem usuários de drogas, mais propensos a serem VIH positivos e a serem empregados do que os nativos, sendo os mesmos mais propensos a usar drogas e álcool. Cabe salientar, nessa conjuntura, que a maioria destes imigrantes é originária de países com o Índice de Desenvolvimento Humano (IDH) menor que Portugal (PAULINO, 2016). O estudo de Redondo (2017), relativamente aos portugueses vivendo em outros países, apontou que os mesmos provavelmente contraíram a TB nos países de acolhimento, todavia, somente realizaram testes para detecção de TB no seu país de origem. Ainda segundo os autores, este fato coloca em perspectiva os desafios da identificação de contatos que deixaram o país, a qual se faz necessário uma rede de comunicação mais efetiva entre as nações a fim de evitar a disseminação da TB e suas complicações.

Sabendo-se do impacto da infecção pelo VIH e a importância do rastreio entre doente com TB e viceversa, verificou-se que a partir da descentralização dos cuidados de saúde houve maior adesão ao teste de TB, contudo, ter doença definidora de SIDA e ter mais idade mostrou-se um fator determinante para a não adesão ao tratamento (Viveiros et al., 2013). Os casos de soropositividade para VIH entre indivíduos com idade acima de 50 anos apresentou crescimento no país, apesar do número de notificações de infecção por VIH ter diminuído (REINA, 2015).

Face ao envelhecimento da população portuguesa, a DM, particularmente a Tipo 2, apresenta maior relação com esta parcela da sociedade em países desenvolvidos como Portugal (CORDEIRO DA COSTA, 2016). Contudo, o mesmo estudo afirma que a incidência de TB está presente em pessoas abaixo da idade 
de aposentadoria, logo, a prevalência de DM entre doentes com TB é baixa no país.

Outro resultado relativo ao grupo etário é o crescimento da taxa de soropositividade para VIH entre indivíduos com idade acima de 50 anos, apesar do número de notificações de infecção por VIH ter diminuído (VIVEIROS, 2013; REINA, 2015). Fator este provavelmente decorrente de práticas sexuais desprotegidas por falsas crenças de não estarem expostos ao VIH como os mais jovens, além disso, a convição de alguns profissionais de saúdede que as pessoas com mais idade são "seres assexuados" conduz a um diagnóstico tardio desta condição clínica (ALENCAR; CIOSAK, 2016).

Contrastando com os indivíduos mais velhos, o estudo de Ladeira et al. (2014) sobre a TB infantil relata os grandes desafios no que diz respeito à confirmação de diagnóstico. As técnicas utilizadas em adultos apresentarem baixa sensibilidade em crianças e a confirmação bacteriológica nem sempre é possível. Desse modo, é comum que o tratamento seja iniciado sem o isolamento da micobactéria, sendo baseado no quadro clínico e radiológico, positividade do teste tuberculínico e no contato com adulto com TB (CANO, 2017).

Em relação à identificação dos contactos, considerada como segunda prioridade nos programas de prevenção e controle da TB, mostrou-se limitada a familiares e co-habitantes, excluindo colegas de trabalho (JOSAPHAT, 2014). Fato este que diverge das recomendações da Comissão de Trabalho de Tuberculose da Sociedade Portuguesa de Pneumologia (2007), uma vez que a relação continuada, frequente e próxima dos portadores de TB ativa com colegas de trabalho coloca em risco a saúde dos demais, além disso, quando oslocais de trabalho exijam contacto físico e/ou possuam más condições são propícios para aumentar a infecção. O estudo de Mendes et al. (2013) apontou que os fatores de risco para resultados positivos no teste de Infecção Latente por TB (ILTB) foram: contacto com idade avançada, ser cohabitante,o doente possuir cultura positiva de expectoração e a duração dos sintomas.

Em relação aos moradores de rua, a incidência de TB foi cinco vezes maior que na população geral entre 2008 e 2014, ficando evidente a vulnerabilidade deste grupo à TB. Ainda segundo o estudo de Dias et al. (2017), estes doentes ao apresentarem idade mais elevada, maiores índices de VIH e uso drogas injetáveis, a adesão ao tratamento torna-se comprometida. Tais características demonstram a necessidade de ações de saúde voltadas para garantir uma atenção integral a estes indivíduos (BRASIL, 2012).

Concernente ao tratamento, ser do sexo masculino, ser seropositivo para o VIH e ter idade acima dos 40 anos foram fatores para abandono de terapêutica e posterior tratamento, resultados estes que convergem com os demais estudos citados anteriormente (PACHECO, 2015). Além disso, a taxa de sucesso foi maior em indivíduos sem tratamento prévio como evidenciado em vários outros estudos (SENGUL, 2015; BERHE, ENQUSELASSIE; ASEFFA, 2012; MUÑOZ-SELLART, 2010).

Ao submeterem-se ao tratamento, os indivíduos estão propensos aos efeitos adversos provocados pelos fármacos. Um estudo aponta que a hepatotoxicidade, rash cutâneo e outras manifestações como toxicidade ocular, intolerância gastrointestinal e angioedema como efeitos adversos mais frequentes (CASTRO, 2015).

Em relação a sílicotuberculose, o estudo de Melo et al. (2016) aponta, novamente, o sexo masculino, idade mais avançada, presença de TB pulmonar ou pleural como fatores de risco para a condição, cujo tratamento é mais prolongado (média de 3 meses mais longo que o regime padrão) e a mortalidade maior. Um dado pertinente do estudo diz respeito ao diagnóstico ser unicamente sustentado pelo exame cultural, demonstrando a dificuldade do diagnóstico de sílicotuberculose.

\section{TUBERCULOSE MULTIRRESISTENTE}

Os casos de desenvolvimento de resistência a fármacos antituberculosos foram associados a presença de infecção pelo VIH, a qual também eleva os índices de mortalidade, e o tratamento prévio foi identificado como o fator de risco mais forte (MACEDO et al., 2012; OLIVEIRA et al., 2013). Um estudo relatou que o tratamento incompleto, hospitalização por tratamento de TB e reação adversa foram os desfechos 
relacionados ao tratamento prévio de doentes com tuberculose multirresistente (TBMR) (RIFAT, 2015).

Observou-se uma alta proporção de resistência a pirazinamida entre os casos de TBMR, sendo este fármaco utilizado tanto no regime da TB sensível quanto nos casos de resistência. Uma pesquisa aponta que nas cepas resistentes a Pirazinamida houve a resistência conjunta com a Rifampicina e a Isoniazida, enfatizando a necessidade da realização do teste de sensibilidade antes do tratamento e, principalmente, em casos de retratamentos (RIBEIRO; MAGALHÃES; MAGALHÃES, 2012; EUFRÁSIO, 2017).

\section{Testes de RASTREio EM PRofissionais de SAÚde}

Foi observado que os testes de detecção não foram oferecidos para a maioria dos profissionais de saúde, indicando insuficiência nas medidas institucionais de controle da TB. Ainda, encontrou-se baixo nível de conhecimento dos trabalhadores acerca da epidemiologia e patogenia da doença, tendo como consequência a resistência dos mesmos à realização dos testes por desinformação (MEIRELES, 2014). Em contraste, osdados da pesquisa realizada por Torres Costa et al. (2011) indicaram um ónus de 129,8 por 100.000 profissionais de saúde, ressaltando a importância da realização de testes de detecção em profissionais de saúde, uma vez que o risco de TB ocupacional é conhecido.

\section{Conclusão}

A literatura portuguesa apresenta relevante produção científica acerca da TB, enfatizando questões sociais, ambientais e económicas particulares de cada região do país. Os resultados são diversificados no que concerne à temática, fornecendo um panorama sobre a realidade nacional e, sob perspectiva de intervenções de controle, podem ser úteis para melhoria de medidas tanto dos programas de luta contra a TB, quanto das outras esferas organizacionais responsáveis por fornecer ações de saúde, sendo as mesmas essenciais para gerar impactos positivos de qualidade na saúde da população.

Salienta-se que as evidências encontradas podem servir de fomento para futuras investigações serem realizadas, principalmente no âmbito da gestão para melhor compreensão da efetividade das políticas de saúde voltadas à temática no país.

\section{Referências}

ALENCAR, R.A.; CIOSAK, S. I. AIDS in the elderly: reasons that lead to late diagnosis. Revista Brasileira de Enfermagem, Brasília, v. 69, n. 6, p. 1076-81, 2016.

AREIAS, C.; BRIZ, T.; NUNES, C. Pulmonary tuberculosis space-time clustering and spatial variation in temporal trends in Portugal, 2000-2010: an updated analysis. Epidemiology \& Infection, United Kingdom, v. 143, n. 15, p. 3211-3219, 2015.

BERHE, G.; ENQUSELASSIE, F.; ASEFFA, A. Treatment outcome of smear-positive pulmonary tuberculosis patients in Tigray Region, Northern Ethiopia. BMC Public Health, London, v. 12, n. 1, p. 537, 2012.

BRAS, A.; GOMES, D.; FILIPE, P.; SOUSA, B.; NUNES, C. Trends, seasonality and forecasts of pulmonary tuberculosis in Portugal. The International Journal of Tuberculosis and Lung Disease, Paris, v. 18, n. 10, p. 1202-1210, 2014.

BRASIL. Ministério da Saúde. Manual sobre o cuidado à saúde junto à população em situação de rua. Brasília, Brasil: Autor, 2012. Disponivel em: http://189.28.128.100/dab/docs/publicacoes/geral/ manual_cuidado_populalcao_rua.pdf 
CANO, A.; ROMANELI, M.; PEREIRA, R.; TRESOLDI, A. Tuberculose em pacientes pediátricos: como tem sido feito o diagnóstico? Revista Paulista de Pediatria, São Paulo, v. 35, n. 2, p. 165-170, 2017.

CASTRO, A.; MENDES, M.; FREITAS, S.; ROXO, P. Incidence and risk factors of major toxicity associated to first-line antituberculosis drugs for latent and active tuberculosis during a period of 10 years. Revista Portuguesa de Pneumologia (English Edition), Lisboa, v. 21, n. 3, p. 144-150, 2015.

CORDEIRO, J. C.; OLIVEIRA, O.; BAÍA, L.; GAIO, R., CORREIA-NEVES, M.; DUARTE, R. Prevalence and factors associated with diabetes mellitus among tuberculosis patients: a nation wide cohort. European Respiratory Journal, Copenhagen, v. 48, n. 1, p. 264-268, 2016.

COUCEIRO, L.; SANTANA, P.; NUNES, C. Pulmonary tuberculosis and risk factors in Portugal: a spatial analysis. The International Journal Of Tuberculosis And Lung Disease, Paris, v. 15, n. 11, p. 1445-1455, 2011.

DIAS, M.; GAIO, R.; SOUSA, P.; ABRANCHES, M.; GOMES, M.; OLIVEIRA, O. ET AL. Tuberculosis among the homeless: should we change the strategy?. The International Journal of Tuberculosis and Lung Disease, Paris, v. 21, n. 3, p. 327-332, 2017.

EUFRÁSIO, R.; ALCOBIA, M.; CORREIA, L. Pulmonary tuberculosis epidemiology in Coimbra’s District (2000-2011): Information is essential to understand high risk groups. Revista Portuguesa de Pneumologia (English Edition), Lisboa, v. 22, n. 4, p. 245-247, 2016.

EUFRÁSIO, R.; ALCOBIA, M.; CORREIA, L. Pulmonary tuberculosis: Resistance pattern to first line antituberculosis drugs in the Coimbra District, 2000-2011. Revista Portuguesa de Pneumologia (English Edition), Lisboa, v. 23, n. 5, p. 300-302, 2017.

EUROPEAN CENTRE FOR DISEASE PREVENTION AND CONTROL. Tuberculosis surveillance and monitoring in Europe 2018 - 2016 data. Stockholm, Sweden: Author, 2018 Disponível em: https:// ecdc.europa.eu/sites/portal/files/documents/ecdc-tuberculosis-surveillance-monitoring-Europe-201819mar2018.pdf

EUROPEAN CENTRE FOR DISEASE PREVENTION AND CONTROL. Tuberculosis. Annual epidemiological report for 2016. Stockholm, Sweden: Author, 2018. Disponível em: https://ecdc.europa. eu/sites/portal/files/documents/AER_for_2016-tuberculosis.pdf

FRANCO, I.; SOUSA, P.; GOMES, M.; OLIVEIRA, A.; GAIO, A.; DUARTE, R. Social profile of the highest tuberculosis incidence areas in Portugal. Revista Portuguesa de Pneumologia (English Edition), Lisboa, v. 22, n.1, p. 50-52, 2016.

GUIMARÃES, M.; OLIVEIRA, O.; TEIXEIRA, C.; GAIO, A.; DUARTE, R. Delay in the diagnosis of tuberculosis. Revista Portuguesa de Pneumologia, Lisboa, 2015.

JOSAPHAT, J.; GOMES DIAS, J.; SALVADOR, S.; RESENDE, V.; DUARTE, R. TUBERCULOSIS: Which patients do notidentify their contacts?. Revista Portuguesa de Pneumologia, Lisboa, v. 20, n. 5, p. 242 $247,2014$. 
Maia et al.

LADEIRA, I.; CORREIA, A.; DIAS, J.; GAIO, R.; CARVALHO, I., CARVALHO, A ET AL. Confirming the diagnosis of tuberculosis in children in Northern Portugal. The International Journal of Tuberculosis and Lung Disease, Paris, v. 18, n. 5, p. 531-533, 2014.

MACEDO, R.; ANTUNES, A.; VILLAR, M.; PORTUGAL, I. Multidrug and extensively drug-resistant tuberculosis in Lisbon and Vale do Tejo, Portugal, from 2008 to 2010. International Journal of Mycobacteriology, v. 1, n. 3, p. 131-136, 2012.

MEIRELES, J.; GAIO, R.; DUARTE, R. Factors influencing tuberculosis screening in healthcare workers in Portugal. European Respiratory Journal, Copenhagen, v. 45, n.3, p.834-838, 2014.

MELO, V.; BAÍA, L.; GAIO, A. R.; DUARTE, R. Silicosis, tuberculosis time bomb?.Revista Portuguesa de Pneumologia (English Edition), Lisboa, v. 22, n. 6, p.355-357, 2016.

MENDES, M.; GAIO, R.; REIS, R.; DUARTE, R. Contact screening in tuberculosis: can we identify those with higher risk?. European Respiratory Journal, Copenhagen, v. 41, n. 3, p. 758-760, 2013.

MOLINA, E. P.; HAPPEL, K. I.; ZHANG, P.; KOLLS, J. K.; NELSON, S. Focus on: alcohol and the immune system. Alcohol \& Health, Rockville, v. 33, n. 1, p. 97-108, 2010.

MOHER, D.; LIBERATI, A.; TETZLAFF, J.; ALTMAN, D. G.; The PRISMA Group Preferred Reporting Items for Systematic Reviews and Meta-Analyses: The PRISMA Statement. PLoS Med, v.6, n.7, p. e1000097, 2009.

MUÑOZ-SELLART, M.; CUEVAS, L. E.; TUMATO, M.; MERID, Y.; YASSIN, M. A.Factors associated with poor tuberculosis treatment outcome in the Southern Region of Ethiopia. International Journal Against Tuberculosis and Lung Disease, Paris, v. 14, n. 8, p. 973-979, 2010.

OLIVEIRA, O.; GAIO, R.; VILLAR, M.; DUARTE, R. Predictors of treatment outcome in multidrugresistant tuberculosis in Portugal. European Respiratory Journal, Copenhagen, v. 42, n. 6, p. 1747-1749, 2013.

PACHECO, C.; SILVA, E.; OLIVEIRA, O.; CARVALHO, A.; CORREIA, A.; DUARTE, R. Tuberculosis retreatment in Northern Portugal. Revista Portuguesa de Pneumologia (English Edition), Lisboa, v. 21, n. 3, p. 166-168, 2015.

PAUliNO, J.; MARTINS, A.; MACHADO, M.; GOMES, M.; GAIO, A.; DUARTE, R. Tuberculosis in native- and foreign-born populations in Portugal. The International Journal of Tuberculosis and Lung Disease, Paris, v. 20, n. 3, p. 357-362, 2016.

PORTUGAL. Ministério da Saúde. Direção-Geral da Saúde. Programa Nacional de Luta Contra a Tuberculose. Lisboa, Portugal: Direção Geral da Saúde, 1995.

PORTUGAL. Ministério da Saúde. Direção-Geral da Saúde. Programa Nacional para a Infeção VIH, Sida e Tuberculose 2017. Lisboa: Direção Geral da Saúde, 2017. 
RABAHI, M. F. Tuberculose e Tabagismo. Revista Pulmão RJ, Rio de Janeiro, v. 21, n. 1, p. 46-49, 2012.

REDONDO, M.; CARVALHO, C.; CORREIA, A.; DUARTE, R. Tuberculosis Among Portuguese Living Abroad. Archivos de Bronconeumología (English Edition), v. 53, n. 5, p. 279-280, 2017.

REINA, S.; SILVA, C.; GAIO, A.; CORREIA, A.; DUARTE, R. HIV screening in tuberculosis patients in the northern region of Portugal. The International Journal of Tuberculosis and Lung Disease, Paris, v. 19, n. 12, p.1554-1555, 2015.

RIBEIRO, L.; MAGALHÃES, V.; MAGALHÃES, M. Resistência primária e adquirida à pirazinamida em pacientes com tuberculose pulmonar atendidos em um hospital de referência no Recife. Jornal Brasileiro de Pneumologia, Brasília, v. 38, n. 6, p. 740-747, 2012.

RIFAT, M.; HALL, J.; OLDMEADOW, C.; HUSAIN, A.; HINDERAKER, S.; MILTON, A. Factors related to previous tuberculosis treatment of patients with multidrug-resistant tuberculosis in Bangladesh. BMJ Open, v. 5, n. 9, 2015. doi: 10.1136/bmjopen-2015-008273.

SAN PEDRO, A.; MAGALHÃES DE OLIVEIRA, R. Tuberculose e indicadores socioeconômicos: revisão sistemática da literatura. Revista Panamericana De Salud Pública, Washington, v. 33, n. 4, p. 294-301, 2013.

SENGUL, A.; AKTURK, U.; AYDEMIR, Y.; KAYA, N.; KOCAK, N.; TASOLAR, F. Factors affecting successful treatment outcomes in pulmonary tuberculosis: a single-center experience in Turkey, 2005-2011. The Journal of Infection in Developing Countries, Sassari, v. 9, n.8, p. 821, 2015.

SOCIEDADE PORTUGUESA DE PNEUMOLOGIA. Tratamento da tuberculose latente: revisão das normas, 2006. Lisboa: Sociedade Portuguesa de Pneumologia, 2007.

SOUSA, P.; OLIVEIRA, A.; GOMES, M.; GAIO, A.; DUARTE, R. Longitudinal clustering of tuberculosis incidence and predictors for the time profiles: the impact of HIV. The International Journal of Tuberculosis and Lung Disease, Paris, v. 20, n. 8, p. 1027-1032, 2016.

TORRES, J. C.; SILVA, R.; RINGSHAUSEN, F.; NIENHAUS, A. Screening for tuberculosis and prediction of disease in Portuguese healthcare workers. Journal of Occupational Medicine and Toxicology, London, v. 6, n. 1, p. 19, 2011.

VIVEIROS, F; MOTA, M.; BRINCA, P.; CARVALHO, A.; DUARTE, R. Adesão ao rastreio e tratamento da tuberculose em doentes infetados com o vírus da imunodeficiência humana. Revista Portuguesa de Pneumologia, Lisboa, v.19, n. 3, p.134-138, 2013.

WHITTEMORE, R.; KNAFL, K. The integrative review: updated methodology. Journal of Advanced Nursing, Oxford, v. 52, n. 5, p. 546-553, 2005.

WORLD HEALTH ORGANIZATION. Global tuberculosis report 2016. Geneva: Switzerland: Author, 2016. Disponível em: http://apps.who.int/medicinedocs/documents/s23098en/s23098en.pdf 